\title{
Facteurs déterminant la fréquence et la sévérité de la mucosite buccale chez les patients subissant une autogreffe de cellules souches
}

\author{
Par Prisco T. Salvador
}

\begin{abstract}
Abrégé
Cette étude descriptive rétrospective portait sur la fréquence de la mucosite buccale et examinait l'incidence de certaines variables sur le développement de la mucosite buccale chez les patients subissant une autogreffe de cellules souches. La mucosite buccale est apparue chez $90 \%$ des patients, tandis que 53,57\% ont souffert d'ulcérations. En moyenne, la mucosite buccale apparaissait le 7 e jour, perdurait 6 jours et se résorbait le $13^{e}$ jour suivant le début de la chimiothérapie. Des bains de bouche au bicarbonate de soude et des collutoires pour la mucosite constituaient les interventions les plus souvent pratiquées; 72,92 \% de ces interventions étaient utilisées à titre de prévention secondaire. La mucosite buccale entretenait un rapport significatif avec le diagnostic (lymphome), la chimiothérapie (étoposide en association avec melphalan) et le niveau de prévention (secondaire). Le diagnostic (lymphome), la chimiothérapie (étoposide en association avec melphalan), la concentration sérique de créatinine (pic) et le niveau de prévention (secondaire) constituaient des variables prédictives indépendantes de la mucosite buccale.
\end{abstract}

\section{Introduction}

La mucosite buccale est l'un des effets secondaires douloureux de la chimiothérapie à haut dosage employée à titre de régime de conditionnement dans les autogreffes de cellules souches (ASCT) les plus fréquemment observés. Les symptômes peuvent apparaître dans la semaine suivant le début de la chimiothérapie chez les patients subissant une ASCT. L'incidence de la mucosite buccale dans le cadre des greffes de cellules souches hématopoïétiques est estimée à $80 \%$ (National Cancer Institute, 2002).

On a mis en évidence certains facteurs de risque aggravant de la mucosite buccale consécutive à une ASCT (Berger et Eilers, 1998; Rapoport et al., 1999), répartis en facteurs relatifs au patient (p. ex. type de malignité, hygiène buccale durant le traitement, âge, état rénal) et en facteurs relatifs au traitement (p. ex. type de chimiothérapie, radiothérapie globale).

Au cours de la dernière décennie, la mucosite buccale a fait l'objet d'un grand nombre d'études et d'articles soulignant l'importance et les aspects bénéfiques d'une harmonisation des pratiques d'hygiène buccale (Larson et al., 1998; Yeager, Webster, Crain, Kasow et McGuire, 2000; Stricker et Sullivan, 2003). En outre, la mise en œuvre de directives, de protocoles et de normes de santé buccale basés sur les résultats pourrait réduire la fréquence des cas de mucosite buccale ou d'en atténuer la sévérité.

En l'absence de littérature sur la prévention et la gestion optimales de la mucosite buccale, il est important que les recherches sur le phénomène de la prévention de la mucosite buccale se poursuivent, d'où cette étude visant à documenter la fréquence de la mucosite buccale et à cerner les facteurs influant sur son développement chez les patients ayant subi une ASCT. Cette étude explorait les questions suivantes: (1) Quelle est l'incidence de la mucosite buccale chez les patients subissant une ASCT?; (2) Quelles sont les caractéristiques de l'apparition, de la phase aiguë et de la résolution de la mucosite buccale chez les patients ayant reçu une ASCT?; (3) Quels sont les modes d'intervention et les niveaux de prévention en tant qu'intervention reconnue qui permettent de prévenir la mucosite buccale?; enfin (4) l'âge, le sexe, le diagnostic, la chimiothérapie, la concentration sérique en créatinine, l'indice de masse corporelle et le niveau de prévention en tant qu'intervention reconnue ont-ils un effet sur le développement de la mucosite buccale chez les patients subissant une ASCT?

\section{Méthodologie}

La documentation de la fréquence de la mucosite buccale et la détermination des facteurs du développement de la mucosite buccale chez les patients ayant subi une ASCT ont été élaborées en utilisant une méthodologie de recherche descriptive et rétrospective. Les données provenaient de 140 dossiers de patients subissant une ASCT admis dans un hôpital universitaire du sud de l'Ontario, au Canada, en 2001 et en 2002. Ces derniers devaient répondre aux critères de sélection suivants: (a) adulte de plus de 18 ans, présentant un diagnostic de myélome multiple (MM), de maladie de Hodgkin ou de lymphome non hodgkinien (LNH); (b) utilisation de facteurs de croissance des colonies de granulocytes (GCSF) dans le cadre du traitement; (c) type de chimiothérapie (melphalan ou étoposide et melphalan).

Avant de procéder à la collecte des données, le chercheur a reçu l'approbation écrite du comité d'éthique de la recherche (CER) de l'hôpital pour réaliser une analyse rétrospective des dossiers à des fins scientifiques. Après avoir pris connaissance de l'approbation du CER, le directeur du programme d'ASCT a fourni au chercheur une liste complète de noms de patients pour les besoins de l'étude.

Le chercheur a lui-même mis au point l'instrument de collecte des données nécessaire. Le contenu a été révisé par deux oncologues médicaux et deux infirmières en oncologie, afin de s'assurer de la consistance interne, de la validité et de la fidélité de l'instrument. L'examen de chaque dossier qui permettait de remplir le formulaire de collecte a requis approximativement 30 minutes au chercheur. $\mathrm{La}$ confidentialité des données des patients a pu être préservée jusqu'au bout grâce à l'attribution à chaque patient d'un numéro de code connu uniquement du chercheur. Tous les formulaires de collecte de données remplis ont été mis sous clé dans un classeur accessible au seul chercheur et y demeureront pour une période de six ans après cette étude.

L'instrument de collecte des données se divise en trois parties: renseignements démographiques; interventions et niveaux de prévention en tant qu'intervention reconnue; et caractéristiques de l'apparition, de la phase aiguë et de la résolution de la mucosite buccale (variable dépendante). Les renseignements démographiques du patient incluent l'âge, le sexe, le diagnostic, le type de chimiothérapie, la concentration sérique en créatinine (niveau de base/pic), et l'indice de masse corporelle (IMC) (variables indépendantes). Les interventions comprennent les agents de soins de la bouche utilisés pour prévenir l'apparition ou limiter la sévérité des signes et des symptômes de la mucosite buccale. Les niveaux de prévention en tant qu'intervention reconnue (une variable indépendante) font référence aux préventions primaire et secondaire décrites dans le modèle des systèmes de Neuman (1995). Une intervention y est appelée prévention primaire lorsqu'elle est pratiquée avant l'apparition des signes et des symptômes de la mucosite buccale. Une intervention est dite secondaire lorsqu'elle est pratiquée après l'apparition de ces signes et symptômes.

Prisco T. Salvador, $R N, M S c N$, Infirmier de chevet, Clinical Teaching Unit, Princess Margaret Hospital, Toronto, Ontario. 
Pour déterminer l'apparition, la phase aiguë et la résolution de la mucosite buccale chez chaque patient, une ligne de temps représentant trois phases d'ASCT avait été définie: pré-transplantation, 4 à 1 jour(s) avant; transplantation, jour 0 ; et post-transplantation, 1 à 12 jours après . Une chimiothérapie à haut dosage (CHD) avait été administrée aux patients présentant un diagnostic de maladie de Hodgkin et LNH les 4 ou 3 e jours précédant la greffe, et le $2^{\mathrm{e}}$ jour avant pour les patients diagnostiqués de MM. Le jour 0, les patients avaient reçu leurs propres cellules souches ou leur propre moelle osseuse cryoconservée(s) après le prélèvement. Dans les 12 jours suivant la greffe, les patients devaient s'attendre à des périodes de myélosuppression et de neutropénie sévère avant que leur numération globulaire ne retrouve des valeurs plus proches de la normale. Le développement de la mucosite buccale chez les patients ayant eu une ASCT - apparition, phase aiguë et résolution - peut survenir à tout moment durant les phases de pré-transplantation, de transplantation ou de post-transplantation. Le degré d'apparition, de phase aiguë et de résolution de la mucosite buccale chez chaque patient a été mesuré suivant le barème des grades de la mucosite buccale défini par l'Organisation mondiale de la santé (1979): 0 - aucun ou normal; I - érythème ou gêne légère; II - érythème, œdème ou ulcères douloureux; III - érythème, œdème ou ulcères douloureux, mais impossibilité de manger; IV - le patient a besoin d'une assistance parentérale ou entérale.

On a fait appel à la statistique descriptive, à la distribution statistique et aux mesures de tendance centrale (médiane et moyenne) pour établir les renseignements démographiques des patients et d'autres variables de l'étude: incidence, apparition, phase aiguë et résolution de la mucosite buccale; et interventions et niveaux de prévention en tant qu'intervention reconnue. Pour examiner l'impact de chaque caractéristique sur le développement de la mucosite buccale, on a eu recours à l'analyse univariée. Le test du chi carré et le modèle logistique à variable unique ont été respectivement employés pour les variables nominales et continues. Ensuite, un modèle de régression logistique multiple a été appliqué pour dégager les facteurs de risque indépendants. La relation entre la variable prédictive et la mucosite buccale était significative pour $\mathrm{p}<0,05$.

Tableau 1: Relation entre les variables prédictives et la fréquence de la mucosite buccale

\begin{tabular}{|c|c|c|c|}
\hline Variables & Catégories & $\begin{array}{c}\% \text { de } \\
\text { mucosite } \\
\text { buccale }\end{array}$ & $\begin{array}{c}\text { Valeur } \\
\text { prédictive }\end{array}$ \\
\hline$\hat{A} g e$ & $\begin{array}{l}<50 \text { ans } \\
>=50\end{array}$ & $\begin{array}{l}47,06 \\
55,66\end{array}$ & 0,3815 \\
\hline Sexe & $\begin{array}{l}\text { Homme } \\
\text { Femme }\end{array}$ & $\begin{array}{l}41,00 \\
60,71\end{array}$ & 0,1665 \\
\hline $\begin{array}{l}\text { Protocole } \\
\text { de chimio }\end{array}$ & $\begin{array}{l}\text { Melphalan } \\
\text { Étoposide/melphalan }\end{array}$ & $\begin{array}{l}43,30 \\
76,74\end{array}$ & $0,0003 *$ \\
\hline Diagnostic & $\begin{array}{l}\text { Myélome multiple } \\
\text { Lymphome } \\
\text { (m. de H./LNH) }\end{array}$ & $\begin{array}{l}43,30 \\
76,74\end{array}$ & $0,0003^{*}$ \\
\hline $\begin{array}{l}\text { Concentration } \\
\text { sérique de } \\
\text { créatinine }\end{array}$ & $\begin{array}{l}\text { Niveau de base } \\
\text { Pic }\end{array}$ & & $\begin{array}{l}0,5701 \\
0,0743\end{array}$ \\
\hline $\begin{array}{l}\text { Indice de } \\
\text { masse corp. }\end{array}$ & & & 0,0764 \\
\hline $\begin{array}{l}\text { Niveau de } \\
\text { prévention }\end{array}$ & $\begin{array}{l}\text { Primaire } \\
\text { Secondaire }\end{array}$ & $\begin{array}{l}41,67 \\
62,50\end{array}$ & $0,0144 *$ \\
\hline$e: * \mathrm{p}$ &, 05 & & \\
\hline
\end{tabular}

\section{Résultats}

L'étude a été menée sur un échantillon de 140 patients subissant une ASCT, dans une tranche d'âge de 19 à 72 ans, avec un âge moyen de 54,96 ans. On constatait la présence d'un plus grand nombre d'hommes (84) que de femmes (56) ainsi qu'une prépondérance de patients présentant un diagnostic de MM (97) et suivant un traitement à base de melphalan (43).

Une mucosite buccale (grades I - IV) s'est développée chez 126 des 140 patients, soit un taux d'incidence de $90 \%$. Les 14 patients restants $(10 \%), 13$ patients atteints de MM et 1 patient atteint de la maladie de Hodgkin, n'ont présenté aucune manifestation de mucosite buccale durant leur traitement. Sur les 126 patients $(90 \%)$ qui avaient une mucosite buccale, 73,02 \% ont vu apparaître les signes et symptômes de la mucosite buccale durant les 7 jours suivant le début de la CHD. Les jours de l'apparition s'échelonnent de 3 à 10, avec une médiane de 7 et une moyenne de 6,63. La phase aiguë de la mucosite buccale (degrés I - IV) a atteint son niveau maximum et perduré pendant 6 jours en moyenne, avec une plage de 2 à 10 jours, une médiane de 6 et une moyenne de 6,26. Une mucosite buccale allant de modérée à plus sévère (degrés II - IV) est survenue dans 53,57 \% (75/140) des cas étudiés. Les patients ont présenté les signes et symptômes de la résolution ou de la guérison de leur mucosite buccale entre le $10^{\mathrm{e}}$ et le

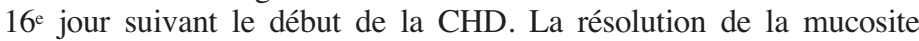
buccale est survenue au plus tard le 14e jour chez 91,27\% des patients.

Des bains de bouche au bicarbonate de soude et des collutoires pour la mucosite (un mélange de nystatine et de xylocaïne visqueuse) constituent les interventions les plus souvent pratiquées aux deux niveaux de prévention en tant qu'intervention reconnue - primaire et secondaire. La prévention primaire a été utilisée à titre d'intervention chez 27,08 \% des patients ayant eu une ASCT. La prévention secondaire a été utilisée chez 72,92\% de ces patients.

Des relations statistiquement significatives avec la mucosite buccale (tableau 1) ont été observées dans la chimiothérapie, le diagnostic et le niveau de prévention pour $\mathrm{p}<=0,05$. L'indice de masse corporelle ( $\mathrm{p}=$ $0,0764)$ et la concentration sérique en créatinine maximale $(\mathrm{p}=0,0743)$ n'avaient qu'une faible portée. L'âge et le sexe n'avaient aucune importance statistique. Le résultat de l'analyse théorique (tableau 2) suggère que le taux de créatinine (maximum) $(\mathrm{p}=0,0436)$, le diagnostic et la chimiothérapie $(p=0,0042)$, et le niveau de prévention $(p=0,0181)$

Tableau 2: Modèle de régression logistique multiple des facteurs influant sur la fréquence et sévérité de la mucosite buccale chez les patients ayant subi une ASCT

\begin{tabular}{|l|c|c|c|c|c|}
\hline Facteur & ddl & Estimation & $\begin{array}{c}\text { Er.- } \\
\text {-type }\end{array}$ & $\begin{array}{c}\text { Wald/ } \\
\text { Chi carré }\end{array}$ & $\begin{array}{c}\text { Valeur } \\
\text { prédictive }\end{array}$ \\
\hline Âge & 1 & 0,0144 & 0,0204 & 0,5037 & 0,4779 \\
\hline Sexe & 1 & 0,5727 & 0,4281 & 1,7898 & 0,1810 \\
\hline $\begin{array}{l}\text { Indice de } \\
\text { masse corp. }\end{array}$ & 1 & $-0,0662$ & 0,0406 & 2,6562 & 0,1031 \\
\hline $\begin{array}{l}\text { Créatinine } \\
\text { (niv. de base) }\end{array}$ & 1 & $-0,0103$ & 0,0137 & 0,5633 & 0,4529 \\
\hline $\begin{array}{l}\text { Créatinine } \\
\text { (pic) }\end{array}$ & 1 & 0,0283 & 0,0140 & 4,0701 & $0,0436^{*}$ \\
\hline Chimiothérapie & 1 & 1,4712 & 0,5141 & 8,1911 & $0,0042^{*}$ \\
\hline Diagnostic & 1 & 1,4712 & 0,5141 & 8,1911 & $0,0042^{*}$ \\
\hline $\begin{array}{l}\text { Prévention } \\
\text { (niveau) }\end{array}$ & 1 & 0,9356 & 0,3957 & 5,5908 & $0,0181 *$ \\
\hline \begin{tabular}{l} 
Note: *p $<=0,05$ \\
\hline
\end{tabular}
\end{tabular}


étaient des facteurs de risque indépendants de la mucosite buccale. Les patients ayant un taux de concentration en créatinine maximal plus élevé, diagnostiqués d'un lymphome (maladie de Hodgkin/LNH), traités à l'étoposide et au melphalan, et faisant l'objet d'une prévention secondaire en tant qu'intervention reconnue, avaient le plus de chances de développer une mucosite buccale allant de modérée à plus sévère.

\section{Discussion}

L'étude descriptive rétrospective portant sur 140 patients traités par CHD plus ASCT a évalué l'incidence de la mucosite buccale (degrés I IV) à $90 \% ; 53,57 \%$ des patients ont souffert de mucosite buccale allant de modérée à plus sévère (degrés II - IV). L'apparition de la mucosite buccale survenait le 7e jour, sa phase aiguë (degrés II - IV) atteignait son seuil maximum et perdurait pendant 6 jours, et se résorbait le $13^{\mathrm{e}}$ jour après le début de la CHD. Les signes et symptômes précoces de la mucosite buccale comprenaient les suivants: érythème du voile du palais, de la muqueuse buccale, de la face ventrale de la langue et du plancher de la bouche; il s'agissait des effets toxiques directs de la chimiothérapie sur les cellules basales de la muqueuse buccale (Woo et al., 1993). L'érythème de la muqueuse buccale s'accompagne souvent d'œdèmes et d'ulcérations. Il est intéressant de constater que la phase aiguë de la mucosite buccale était particulièrement sensible pendant le nadir hématologique des patients, caractérisé par une myélosuppression et une neutropénie sévère. En revanche, la résolution de la mucosite buccale coïncide avec la récupération des neutrophiles (Woo et al., 1993).

Tandis que l'incidence totale de la mucosite buccale atteignait $90 \%$ dans cette étude, l'incidence de la mucosite buccale ulcéreuse, estimée à $53,57 \%$, était comparativement moins élevée que dans des études précédentes. Il apparaît dans la littérature que les patients soumis à des régimes de conditionnement pour une greffe de moelle ou de greffe de cellules souches du sang périphérique présentent des incidences de mucosite buccale supérieures. Pour ce qui est des greffes de cellules souches hématopoiétiques, le National Cancer Institute (2002) estimait la fréquence des cas de mucosite buccale à $80 \%$. Woo et al. (1993) faisaient état dans leur étude de la prédominance d'une mucosite buccale ulcéreuse à 76,3 \%. Dans un essai sur échantillon aléatoire et contrôlé comparant l'efficacité de deux soins d'hygiène buccale dans les greffes de moelle osseuse, Borowski et ses collaborateurs (1994) ont observé un développement de mucosite allant de modérée à plus sévère de $89,3 \%$.

Dans cette étude, la résolution de la mucosite buccale survenait le $13^{\mathrm{e}}$ jour après la CHD, soit plus tôt que dans les conclusions de Woo et al. (1993), qui la situaient autour du 18e jour. La différence pourrait être attribuée à l'administration des facteurs de croissance des colonies de granulocytes (GCSF) dans le cadre du traitement de tous les patients constituant cette étude. Selon des études antérieures, les GCSF peuvent favoriser la prolifération des neutrophiles et accélérer la récupération des neutrophiles du sang périphérique. En outre, les GCSF se sont révélés aptes à réduire la fréquence et la sévérité de la mucosite buccale consécutive à la chimiothérapie (Lieschke et al., 1992; Katano, Nakamura, Matsuo, Iyama et Hisatsugu, 1995). Malheureusement, le résultat de cette comparaison sur l'incidence de la mucosite buccale est difficile à interpréter du fait de l'hétérogénéité de la population de patients, des écarts en matière de taille de l'échantillon et de la diversité des protocoles de chimiothérapie utilisés dans les différentes études.

Pour examiner les relations entre l'âge, le sexe, le diagnostic, le type de chimiothérapie, la concentration sérique en créatinine, l'indice de masse corporelle et le niveau de prévention, d'une part, et la mucosite buccale, d'autre part, on a eu recours à des analyses univariées et multivariées. Cette étude a démontré que des variables tel que l'âge, le sexe, l'indice de masse corporelle et la concentration sérique en créatinine en situation de référence n'entretenaient pas de lien significatif avec l'incidence de la mucosite buccale. En dépit du fait que $55 \%$ des sujets de l'étude étaient âgés de plus de 50 ans, cette étude ne confirme pas la tendance à une prédominance et à une sévérité accrues de la mucosite chez les patients plus âgés qui avait été relatée par McCarthy et al., (1998).
Chez les patients plus âgés, la détérioration physiologique de la fonction rénale due au vieillissement peut favoriser l'accroissement de la toxicité chimiothérapique et susciter une plus grande vulnérabilité à la mucosite buccale (Balducci et Mowry, 1992). Étonnamment, $40 \%$ des sujets de l'étude étaient des femmes, et plus de $60 \%$ d'entre elles ont développé une mucosite buccale, mais le sexe ne constituait pas un facteur de risque en terme de statistiques. Les conclusions de cette étude sur le sexe comme facteur de risque viennent corroborer celles de McCarthy et al., (1998).

Dans une étude de Raber-Durlacher et al. (2000), les patients atteints de tumeurs solides et ayant un indice de masse corporelle $(\mathrm{IMC})<20$, étaient légèrement plus prédisposés à la mucosite que ceux ayant un IMC $>20$. Par contraste, l'IMC des patients ayant subi une ASCT était $>20$ et ne laissait entrevoir qu'un effet négligeable $(0,0764)$ sur le développement de la mucosite buccale. La plupart du temps, les patients souffrant de mucosite buccale ulcéreuse n'étaient pas capables de manger ni même de boire pendant une semaine ou plus, à cause de la douleur extrême associée à la déglutition; cette condition pouvait conduire à une mucosite plus sévère due à une déficience de la régénération des muqueuses (Sonis, 1993). Les patients ayant reçu une ASCT qui présentent une mucosite buccale ulcéreuse pourraient bénéficier d'une intervention précoce grâce à une évaluation buccale méticuleuse et à une approche multidisciplinaire.

Cette étude a fait apparaître un lien négligeable entre le pic de concentration sérique en créatinine après la chimiothérapie et la mucosite buccale; c'est aussi une variable prédictive indépendante de la mucosite buccale. Il en ressort que les patients subissant une ASCT dont la concentration sérique en créatinine post-transplant est plus élevée présentent un risque plus important de mucosite buccale sévère. La concentration sérique en créatinine de ces patients devrait donc être suivie quotidiennement, de manière à détecter toute augmentation de niveau, et ceux-ci devraient pouvoir bénéficier d'une intervention précoce.

Le diagnostic, la chimiothérapie et le niveau de prévention étaient liés en permanence avec l'incidence de la mucosite buccale et constituent des prédicteurs indépendants de la mucosite. Ces résultats indiquent que les patients ayant subi une ASCT diagnostiqués d'un lymphome (maladie de Hodgkin,LNH), traités à l'étoposide et au melphalan, et soumis à une prévention secondaire en tant qu'intervention reconnue, présentent un risque plus élevé de contracter une mucosite sévère. L'incidence des complications orales est généralement plus élevée dans les cancers hématologiques que dans les tumeurs solides à cause du degré d'immunosuppression éprouvé par cette population (Raber-Durlacher et al., 2000). À la lumière de ces résultats, les patients ayant eu une ASCT pourraient bénéficier de l'application de la prévention primaire en tant qu'intervention reconnue. Diverses études ont documenté les bienfaits d'une évaluation buccale systématique et de l'adhésion à un protocole de soins buccaux en tant qu'intervention prophylactique pour réduire ou prévenir les cas de mucosite buccale (Beck, 1979; Larson et al., 1998; Dodd et al., 2000).

La méthode de collecte des données utilisée dans cette étude reposait essentiellement sur la documentation des dossiers fournie par les professionnels de différentes disciplines de la santé - infirmières, médecins, algologues et nutritionnistes. Certaines variables ont probablement été sous-déclarées. En outre, les données n'étaient pas présentées à des fins de recherche. Par conséquent, les omissions ou les fautes - potentiellement, de sérieuses sources d'erreur - n'ont pas pu être vérifiées.

Une étude prospective descriptive est actuellement à l'examen, en vue d'évaluer les effets, sur le développement de la mucosite buccale ulcéreuse, de facteurs tels que les soins dentaires préventifs, le diagnostic, les régimes de conditionnement, la pratique d'une hygiène buccale globale et l'utilisation des facteurs de croissance des colonies de granulocytes. L'utilisation d'un outil d'évaluation buccale valide et fidèle s'avèrera une composante importante de cette étude. 


\section{Références}

Balducci, L., \& Mowry, K. (1992). Pharmacology and organ toxicity of chemotherapy in older patients. Oncology, 6, 62-68.

Beck, S. (1979). Impact of a systematic oral care protocol on stomatitis after chemotherapy. Cancer Nursing, 2, 185-199.

Berger, A., \& Eilers, J. (1998). Factors influencing oral cavity status during high-dose antineoplastic therapy: A second data analysis. Oncology Nursing Forum, 25, 1623-1626.

Borowski, B., Benhamou, E., Pico, J.L., Laplanche, A., Margainaud, J.P., \& Hayat, M. (1994). Prevention of oral mucositis in patients treated with high-dose chemotherapy and bone marrow transplantation: A randomized controlled trial comparing two protocols of dental care. Oral Oncology, European Journal of Cancer, 30B, 93-97.

Dodd, M.J., Dibble, S.L., Miaskowski, C., MacPhail, L., Greenspan, D., Paul, S.M., et al. (2000). Randomized clinical trial of the effectiveness of three commonly used mouthwashes to treat chemotherapy-induced mucositis. Oral Surg Oral Med Oral Pathol Oral Radiol Endod, 90(1), 39-47.

Katano, M., Nakamura, M., Matsuo, T., Iyama, A., \& Hisatsugu, T. (1995). Effect of granulocyte colony stimulating factor (G-CSF) on chemotherapy-induced oral mucositis. Surgery Today, 25, 202-206.

Larson, P.J., Miaskowski, C., MacPhail, L., Dodd, M.J., Greenspan, D., Dibble, S.L., et al. (1998). The pro-self mouth aware program: An effective approach for reducing chemotherapy-induced mucositis. Cancer Nursing, 21(4). 263-268.

Lieschke, G., Ramenghi, U., O'Connor, M., Sheridan, W., Szer, J., Morstyn, G. (1992). Studies of oral neutrophil levels in patients receiving G-CSF after autologous marrow transplantation. British Journal of Haematology, 82, 589-95.

McCarthy, G.M., Awde, J.D., Ghandi, H., Vincent, M., \& Kocha, W.I. (1998). Risk factors associated with mucositis in cancer patients receiving 5-fluorouracil. Oral Oncology, 34, 484-490.
National Cancer Institute. (2002). Oral complications of chemotherapy and head/neck radiation (PDQ). Retrieved October 30, 2003, from http://www.cancer.gov/cancerinfo/pdq/supportivecare/oral complications/HealthProfessional

Neuman, B. (1995). The Neuman systems model (3rd ed.). Norwalk, CT: Appleton \& Lange.

Raber-Durlacher, J.E., Weijl, N.I., Abu Saris, M., de Koning, B., Zwinderman, A.H., \& Osanto, S. (2000). Oral mucositis in patients treated with chemotherapy for solid tumours: A retrospective analysis of 150 cases. Supportive Care in Cancer, 8, 366-371.

Rapoport, A.P., Miller Watelet, L.F., Linder, T., Eberly, S., Raubertas, R.F., Lipp, J., et al. (1999). Analysis of factors that correlate with mucositis in recipients of autologous and allogenic stem cell transplants. Journal of Clinical Oncology, 17, 2446-2453.

Sonis, S.T. (1993). Oral complications of cancer therapy. In V.T. De Vita, S. Hellman, \& S.A. Rosenberg (Eds.), Cancer: Principles and practices of oncology (4th ed.) (p. 2385-2394). Philadelphia, PA: Lippincott.

Stricker, C.T., \& Sullivan, J. (2003). Evidence-based oncology oral care clinical practice guidelines: Development, implementation, and evaluation. Clinical Journal of Oncology Nursing, 7(2), 222-227.

Woo, S., Sonis, S.T., Monopoli, M.M., \& Sonis, A.L. (1993). A longitudinal study of oral ulcerative mucositis in bone marrow transplant recipients. Cancer, 72, 1612-1617.

World Health Organization. (1979). Handbook for reporting results of cancer treatment. Geneva, Switzerland: WHO.

Yeager, K.A., Webster, J., Cram, M., Kasow, J., \& McGuire, D.B. (2000). Implementation of an oral care standard for leukemia and transplantation patients. Cancer Nursing, 23, 40-47. 